\title{
Indicações Clínico-Epidemiológicas para Investigação Ecocardiográfica no Período Neonatal. Valor dos Grupos de Risco
}

Lilian dos Santos Rodrigues Sadeck, Renato Azevedo, Alfonso Julio Guedes Barbato, Valdenise M. L. T. Calil, Maria do Rosário Dias de Oliveira Latorre, Cléa Rodrigues Leone, José Lauro Araújo Ramos

São Paulo, SP

Objetivo - Estudo ecocardiográfico em recém-nascidos $(R N)$ de grupos de risco para cardiopatia congênita, a fim de se determinar a prevalência que justifique esse exame no período neonatal.

Métodos - Estudaram-se, de novembro/91 a abril/93, 156 RN do berçário anexo à Maternidade do HC-FMUSP, sendo RN de mães com cardiopatia congênita ou com diabetes mellitus, crianças de muito baixo peso, com malformações extracardíacas ou presença de sinais cardíacos, caracterizados por sopro, cianose ou arritmia, todos submetidos ao ecocardiograma.

Resultados - A prevalência encontrada foi de 21,8\%, superior ao da população geral ( 0,8 a 1,2\%), sendo que a maior entre os grupos, de 40,7\%, ocorreu no grupo de malformações extracardíacas.

Conclusão - Nossos dados justificam a realização de ecocardiograma em $R N$ pertencentes a um destes grupos de risco para cardiopatia congênita.

Palavras-chaves: cardiopatias congênitas, ecocardiografia, recém-nascidos

\section{Clinical-Epidemiologic Indications for Echocardiographic Investigation in the Neonatal Period. Value of Risk Groups}

Purpose - An echocardiographic study was performed in newborns from risk groups, with the aim of to determining prevalence and to evaluate the indications for this test in the neonatal period.

Methods - One hundred fifty six newborns were studied. They were admitted to the Newborn ward of the Department of Obstetric of the HC-FMUSP, in the period of November 91 to April 93, from mothers with congenital heart disease or diabetes, low birth weight newborns, with extracardiac malformations, cardiac signs and/or congenital infections.

Results - The observed prevalence was $21.8 \%$, greater than that of the general population (0.8-1.2\%). The group composed by 27 children with extracardiac abnormalities presented the largest prevalence when compared with the other groups (40.7\%). These data justify the use of echocardiography in high risknewborns for the detection of congenital heart disease.

Key-words: congenital heart diseases, echocardiography, newborn/infant

Arq Bras Cardiol, volume 69 (n 5), 301-307, 1997

Com os recentes avanços ocorridos na assistência perinatal, tem sido possível diminuir a mortalidade neonatal decorrente de condições, como prematuridade, asfixia e infecção. Com isto, vêm aumentando, proporcionalmente, os óbitos decorrentes de malformações congênitas. A incidência de malformações congênitas ${ }^{1,2}$ maiores varia de 2 a

Instituto da Criança Prof. Pedro de Alcântara - Hospital das Clínicas - FMUSP Correspondência: Lilian S. Rodrigues Sadeck - Rua Dr. Augusto de Miranda, 1092/83 - 05026-001 - São Paulo, SP

Recebido para publicação em 19/5/97

Aceito em 27/8/97
$2,5 \%$ do total de nascimentos. Estudo efetuado em nosso meio revelou uma cifra de $1,67 \%{ }^{3}$. Dentre as malformações, um terço é representado pelas cardiopatias congênitas (CC), que são responsáveis por $50 \%$ do total de óbitos por malformações ${ }^{4}$. A maioria dos óbitos decorrente de CC ocorre intra-útero ou no período neonatal.

Manetti e $\mathrm{col}^{5}$, avaliando 46.895 nascidos vivos, na Maternidade de Careggi em Florença, no período de 1975 a 1984, detectaram uma prevalência de CC de 1,23\%. Destes, 22,6\% evoluíram para óbito. No período subseqüente, de 1985-1990, os mesmos autores encontraram 172 recém-nascidos $(\mathrm{RN})$ com CC, sendo que apenas $13,4 \%$ evoluíram para 
óbito, mostrando uma sensível redução da mortalidade nesse período. Provavelmente, esses resultados se devem à evolução das técnicas diagnósticas e da terapêutica disponível, especialmente, ao avanço da ecocardiografia (ECO) e à utilização de prostaglandinas, possibilitando, portanto, o encaminhamento mais precoce dos pacientes a um tratamento cirúrgico, em condições clínicas mais estáveis. Paralelamente a este avanço no diagnóstico das CC houve, também, progresso das técnicas cirúrgicas e dos cuidados pré e pós-operatórios, inclusive em recém-nascidos pré-termo (RNPT), favorecendo ainda mais o prognóstico dessas crianças e aumentando sua sobrevida ${ }^{6}$.

A partir do princípio de que, quanto mais cedo seja feito o diagnóstico da $\mathrm{CC}$, melhor o seu prognóstico, alguns autores vêm estudando o impacto da utilização de ECO fetal, como triagem de $\mathrm{CC}$, em estudos populacionais ${ }^{7,9}$ sobre a evolução deste distúrbio.

Os dados relativos à alta mortalidade por $\mathrm{CC}$, concentrada no $1^{\circ}$ mês de vida, associados à possibilidade de diagnóstico por métodos não invasivos e à realização, com sucesso, de intervenções terapêuticas e correções cirúrgicas, ainda no período neonatal, têm estimulado os neonatologistas a investigar o diagnóstico mais precoce das CC. Tendo em vista esta necessidade, optou-se pela realização de um estudo, com o objetivo de determinar se a prevalência de CC em RN pertencentes a grupos de risco justifica a utilização sistemática da ECO na assistência neonatal desses indivíduos.

\section{Métodos}

Em revisão bibliográfica determinaram-se cinco principais grupos de risco, reconhecidos na literatura para CC: grupo I - filhos de mães com CC ${ }^{10,11}$; grupo II - filhos de mães com diabetes mellitus ${ }^{12-14}$; grupo III - RN com peso $<1500 \mathrm{~g}{ }^{15,16}$, grupo IV - RN portadores de malformações extracardíacas ${ }^{17,18}$ e grupo V - RN com sinais sugestivos de comprometimento cardíaco, caracterizado por sopro, cianose central e/ou arritmia cardíaca $^{19,20}$.

Realizou-se, estudo descritivo, prospectivo, em 156 $\mathrm{RN}$ (pertencentes a um desses grupos), admitidos no berçário anexo à Maternidade do Hospital das Clínicas da Faculdade de Medicina da USP (HC-FMUSP), de novembro/91 a abril/93, determinando-se sua prevalência de CC de cada grupo de risco. Verificaram-se quais as $\mathrm{CC}$ mais freqüentes e suas associações com sexo, cor, peso de nascimento, idade gestacional, adequação do crescimento intra-uterino, idade pós-natal, idade pós-concepcional e sinais cardíacos. Determinaram-se, também, os grupos de maior chance de apresentarem CC.

A inclusão dos RN no grupo I ou grupo II baseou-se nos diagnósticos maternos obtidos nos dados presentes no prontuário materno, por ocasião do parto. O grupo III, constituído por RN com peso $<1500 \mathrm{~g}$ não apresentava nenhuma das características dos demais grupos de risco. No grupo IV foram englobados aqueles que apresentavam malformações maiores, diagnosticadas no período peri- natal. No grupo V só foram classificados aqueles que apresentavam peso de nascimento $>1499 \mathrm{~g}$, com sinais sugestivos de cardiopatia e que não tinham nenhum outro fator associado. A distribuição dos RN de acordo com o grupo de risco é apresentada na tabela I.

Foram estudadas as seguintes variáveis: cardiopatia congênita, definida como uma anormalidade estrutural do coração ou dos grandes vasos, com repercussão na função cardíaca, seja real ou potencial, e que esteja presente desde o nascimento, mesmo que só descoberta mais tardiamente. A persistência de canal arterial (PCA) só é considerada como cardiopatia congênita, se perdurar por mais de 14 dias de vida ${ }^{13}$; peso de nascimento; idade gestacional, baseada na informação materna quanto à data da última menstruação, desde que não diferisse em mais de duas semanas da idade estimada pelo método de Dubowitz. Para a classificação por idade gestacional, utilizou-se o critério da Organização Mundial de Saúde (1961): idade pós-concepcional é a idade gestacional definitiva acrescida das semanas de vida pós-natal. A qualidade do crescimento intra-uterino foi avaliada pela curva de crescimento intra-uterino de $\operatorname{Ramos}^{21}$; idade pós-natal: em dias; sexo: masculino e feminino; cor: branca e não branca; sinais cardíacos, considerando-se como sinais sugestivos de cardiopatia, a presença de sopro, crises de cianose ou alterações do ritmo cardíaco detectado pelo exame clínico.

A CC foi diagnosticada pela ECO, nos RN selecionados, durante o período de internação no berçário. O exame foi realizado em todos os RN, sempre pelo mesmo cardiologista pediátrico, com especialização em ECO, realizado em um aparelho Toshiba ${ }^{\circledR}$ com transdutor setorial de $5 \mathrm{MHz}$. Os RN foram estudados com ECO modo-M, bidimensional, Doppler pulsátil e contínuo e mapeamento do fluxo a cores, utilizando-se as incidências preconizadas na literatura para o período neonatal. Todos os exames foram realizados na Unidade de Radiologia do HC-FMUSP. Os RN eram encaminhados para essa unidade, localizada no mesmo prédio, em incubadoras de transporte e os exames aí realizados, com o RN em posição supina e sem o uso de sedação medicamentosa.

Para cumprir os objetivos deste trabalho, foram montadas tabelas descritivas e de freqüências das variáveis estudadas: cardiopatias congênitas, sexo, peso de nascimento, adequação de peso, idade gestacional, idade pós-natal, idade pós-conceptual e presença de sintomas, utilizando-se o programa EPI-info. Calcularam-se a prevalência de CC em relação ao total dos RN estudados e a prevalência em cada grupo de risco. Verificou-se a freqüência de cada tipo de CC. Compararam-se essas prevalências de $\mathrm{CC}$ e a freqüência de cada tipo de lesão da população estudada com as prevalências e as frequiências encontradas na literatura. Determinou - se a odds ratio (OR) entre os grupos de risco ${ }^{22}$.

Foi feita uma análise bivariada para avaliar a associação da CC com o sexo, cor, peso, crescimento intra-uterino, idade gestacional, idade pós-natal, idade pós-concepcional e sintomas. Avaliou-se, também, a associação das principais cardiopatias encontradas em relação às variáveis men- 


\begin{tabular}{|c|c|c|}
\hline \multicolumn{3}{|c|}{$\begin{array}{l}\text { Tabela I : Distribuição dos recém-nascidos de acordo com o grupo } \\
\text { de risco }\end{array}$} \\
\hline Grupos & Casos $\left(n^{\circ}\right)$ & Porcentagem $(\%)$ \\
\hline Grupo I & 24 & 15,4 \\
\hline Grupo II & 28 & 18,0 \\
\hline Grupo III & 36 & 23,0 \\
\hline Grupo IV & 27 & 17,3 \\
\hline Grupo V & 41 & 26,3 \\
\hline Total & 156 & 100,0 \\
\hline
\end{tabular}

\begin{tabular}{|lccccc|}
\hline \multicolumn{6}{|c|}{ Tabela II - Distribuição dos recém-nascidos, de acordo com o grupo de } \\
risco e presença de cardiopatia congênita (CC)
\end{tabular}

cionadas. A possível significância estatística das associações encontradas nessas análises foi avaliada pelo quiquadrado, com correção de Yates para tabelas $2 \times 2$ ou $2 \times n$ e, quando os valores esperados eram $<5$, foi utilizado o teste exato de Fisher, com o programa Epi-info função Statcalc. Fixou-se o nível de significância em 5\%.

\section{Resultados}

Cardiopatia congênita, diagnosticada através da ECO e segundo a definição adotada, esteve presente em 34 RN desta população de risco, determinando uma prevalência geral de $21,8 \%$. Estudando a prevalência de $\mathrm{CC}$ em cada um dos grupos de risco, verificou-se que esta variou de 10,7\% até $40,7 \%$ (tab. II).

A distribuição dos $156 \mathrm{RN}$, de acordo com o grupo de risco, presença de sinais cardíacos e presença de cardiopatia congênita, encontra-se na tabela III. Analisando todos os $62 \mathrm{RN}$ que apresentaram sinais cardíacos, independentemente de estarem ou não em outros grupos de risco, obteve-se CC em 21 (34\%) RN, dado que difere da prevalência calculada para os $\mathrm{RN}$ do grupo $\mathrm{V}$, constituído por $\mathrm{RN}$ portadores de sinais cardíacos, mas sem outros agravantes, que foi de 26,8\% (tab. II). A análise destes dados sugere que a coexistência de sinais cardíacos e outra indicação para a realização deECO aumentam a prevalência da CC.

Observou-se também na tabela III que, dos $34 \mathrm{RN}$ com CC, 21 apresentavam sinais sugestivos de cardiopatia, enquanto 13 não, tendo sido indicada a exploração ecocardiográfica por pertencerem a outros grupos de risco. Chama a atenção que a ECO não detectou cardiopatia em 41 RN que apresentavam sinais cardíacos.

Através de uma análise bivariada, analisou-se a existência de associação entre a presença de CC e as variáveis estudadas, sexo, cor, peso de nascimento, idade gestacional, adequação do peso para a idade gestacional, idade pós-natal, idade pós- conceptual e a presença de sinais de comprometimento cardíaco.

Não foi verificada associação estatisticamente significante entre a presença de CC e as variáveis estudadas, exceto em relação à presença de sinais cardíacos e sopro cardíaco. Dos $62 \mathrm{RN}$ que apresentavam sinais cardíacos, 21 $(33,8 \%)$ tinham CC diagnosticada através de ECO, e, em relação à presença de sopro cardíaco, dos $47 \mathrm{RN}$ que o apresentavam, em 20 (42,5\%) foi diagnosticado CC.

Calculou-se o valor preditivo positivo e negativo da presença de sopro cardíaco para a cardiopatia congênita. $\mathrm{O}$ valor preditivo positivo foi de $42 \%$ e o negativo, de $87 \%$. Quando determinou-se o valor preditivo positivo do sopro cardíaco, considerando-se apenas os $\mathrm{RN}$ pertencentes ao grupo $\mathrm{V}$, isto é, que apresentavam apenas como indicação de ECO a presença de sinais clínicos, observou-se que este foi de $38 \%$, enquanto o valor preditivo negativo foi de $93 \%$. $\mathrm{E}$, determinando o valor preditivo positivo no grupo de $\mathrm{RN}$ que apresentava, além do sopro cardíaco, outra indicação de ECO, este foi de $48 \%$ e o valor preditivo negativo diminuiu para $86 \%$.

A razão de chance (OR) para cada um dos grupos de risco, em relação aos demais grupos, encontra-se na tabela IV.

É importante observar que nos grupos I, II, III, V, nem a OR, nem o qui-quadrado, mostraram-se estatisticamente significantes, já que estamos comparando a presença de $\mathrm{CC}$ em cada grupo de risco em relação aos RN pertencentes aos outros grupos de risco. No grupo IV, verificou-se que existe uma associação estatisticamente significante entre a presença de malformações extracardíacas e a de CC.E a OR mostra que o RN portador de malformação extracardíaca tem de 1,12 a 7,83 vezes mais chances de ter CC, quando comparado com os demais $\mathrm{RN}$ dos outros grupos de risco. Quando analisaram-se os $\mathrm{RN}$ portadores de sinais cardíacos e com sopro, verificou-se que a chance de terem CC aumentava 3,58 e 5,65 vezes, respectivamente.

Os RN com CC foram distribuídos de acordo com o tipo de lesão específica, classificada conforme o código da Internacional Society of Cardiology ${ }^{23}$ e pelo estudo de Ferencz e col ${ }^{24}$. Encontraram-se 12 casos de comunicação interatrial (CIA), quatro de comunicação interventricular (CIV), seis de PCA, dois de atrioventricularis comunis (AVC), dois de estenose pulmonar (EP), quatro casos de associação, dois de cardiopatia complexa, um de tetralogia de Fallot e, um de situs inversus. Nesta casuística, observase que o tipo de lesão mais freqüente foi o defeito de septo interatrial.

Como foi descrito anteriormente, classificou-se como CIV apenas os casos isolados desta cardiopatia. Nos quatro casos classificados como associação, em dois os achados foram CIV + CIA e, nos outros dois, CIV + CIA + PCA. Al- 


\begin{tabular}{|c|c|c|c|c|}
\hline \multicolumn{5}{|c|}{$\begin{array}{l}\text { Tabela III - Distribuição dos recém-nascidos, de acordo com o grupo de } \\
\text { risco, presença de sinais cardíacos e cardiopatia congênita (CC) }\end{array}$} \\
\hline \multicolumn{5}{|c|}{ Sinais cardíacos } \\
\hline Grupo & Presença de CC & Presente & Ausente & Total \\
\hline \multirow[t]{2}{*}{ I } & Presente & 1 & 4 & 5 \\
\hline & Ausente & 2 & 17 & 19 \\
\hline \multirow[t]{2}{*}{ II } & Presente & 2 & 1 & 3 \\
\hline & Ausente & 1 & 24 & 25 \\
\hline \multirow[t]{2}{*}{ III } & Presente & 3 & 1 & 4 \\
\hline & Ausente & 7 & 25 & 32 \\
\hline \multirow[t]{2}{*}{ IV } & Presente & 4 & 7 & 11 \\
\hline & Ausente & 1 & 15 & 16 \\
\hline \multirow[t]{2}{*}{$\mathrm{V}$} & Presente & 11 & - & 11 \\
\hline & Ausente & 30 & - & 30 \\
\hline Total & & 62 & 94 & 156 \\
\hline
\end{tabular}

\begin{tabular}{|c|c|c|c|c|}
\hline \multicolumn{5}{|c|}{$\begin{array}{l}\text { Tabela IV - Distribuição dos recém-nascidos, de acordo com a } \\
\text { presença de cardiopatia congênita (CC) e o grupo de risco (odds ratio) }\end{array}$} \\
\hline \multirow{2}{*}{$\begin{array}{l}\text { Grupos de } \\
\text { risco }\end{array}$} & \multicolumn{2}{|c|}{ Presença de CC } & \multirow[t]{2}{*}{ Odds Ratio } & \multirow{2}{*}{$\begin{array}{c}\text { Intervalo } \\
\text { de confiança }\end{array}$} \\
\hline & Sim & Não & & \\
\hline I & 5 & 19 & 0,89 & $0,24<\mathrm{OR}<2,74$ \\
\hline II & 3 & 25 & 0,36 & $0,07<\mathrm{OR}<1,31$ \\
\hline III & 4 & 32 & 0,36 & $0,09<\mathrm{OR}<1,14$ \\
\hline IV & 11 & 16 & $2,97 *$ & $1,12<\mathrm{OR}<7,83$ \\
\hline V & 11 & 30 & 1,38 & $0,54<\mathrm{OR}<3,32$ \\
\hline Sinais & 23 & 41 & $3,58 *$ & $1,55<\mathrm{OR}<8,48$ \\
\hline Sopro & 22 & 27 & $5,65 *$ & $2,38<\mathrm{OR}<13,53$ \\
\hline
\end{tabular}

Tabela V - Distribuição dos recém-nascidos com cardiopatia congênita (CC), de acordo com o tipo de cardiopatia e o grupo de risco

\begin{tabular}{|c|c|c|c|c|c|c|c|}
\hline \multicolumn{8}{|c|}{ Grupos de risco } \\
\hline \multirow[t]{2}{*}{ Tipo de CC * } & I & II & III & IV & $\mathrm{V}$ & Total & \\
\hline & $\mathrm{N}^{\mathrm{o}}$ & $\mathrm{N}^{\mathrm{o}}$ & $\mathrm{N}^{\mathrm{o}}$ & $\mathrm{N}^{\mathrm{o}}$ & $\mathrm{N}^{\circ}$ & $\mathrm{N}^{\mathrm{o}}$ & $\%$ \\
\hline CIA & 4 & 1 & 2 & 3 & 2 & 12 & 35,2 \\
\hline CIV & 1 & - & 1 & 1 & 1 & 4 & 11,7 \\
\hline PCA & - & - & 1 & 2 & 3 & 6 & 17,6 \\
\hline $\mathrm{T} 4 \mathrm{~F}$ & - & - & - & - & 1 & 1 & 2,9 \\
\hline AVC & - & - & - & 1 & 1 & 2 & 5,9 \\
\hline EP & - & - & - & 2 & - & 2 & 5,9 \\
\hline Complexa & - & 1 & - & - & 1 & 2 & 5,9 \\
\hline Associação & - & 1 & - & 1 & 2 & 4 & 11,7 \\
\hline Situs inversus & - & - & - & 1 & - & 1 & 2,9 \\
\hline Total & 5 & 3 & 4 & 11 & 11 & 34 & 100 \\
\hline $\begin{array}{l}\text { * Tipo: CIA- } \\
\text { PCA-persistên } \\
\text { estenose pulm }\end{array}$ & $\begin{array}{l}\text { con } \\
\text { cia } \\
\text { onar; }\end{array}$ & & $\begin{array}{l}\text { tria } \\
\text {; AI }\end{array}$ & . & $\begin{array}{l}\text { aicaç } \\
\text { icula }\end{array}$ & $\begin{array}{l}\text { terve } \\
\text { omm }\end{array}$ & $\begin{array}{l}\text { cular; } \\
\text {; EP- }\end{array}$ \\
\hline
\end{tabular}

guns autores classificariam esses casos como CIV complicada, o que somaria oito $(23,5 \%)$ casos de CIV.

Os casos de EP apresentavam gradiente de pressão de $10,5 \mathrm{e} 13 \mathrm{mmHg}$. Nos dois casos classificados como cardiopatia complexa, os achados ecocardiográficos foram: dupla via de saída de ventrículo direito (VD) + PCA + CIA + CIV; $\mathrm{EP}+\mathrm{CIA}+\mathrm{PCA}$. Os dois casos tinham diagnóstico intraútero de cardiopatia, através da ECO fetal e apresentaram sintomatologia, caracterizada por sopro logo após o nascimento.

Na tabela $\mathrm{V}$ encontram-se os tipos de $\mathrm{CC}$ de acordo com os grupos de risco.

Analisando-se a existência de associação entre os três tipos de CC mais freqüentes e as variáveis estudadas: sexo, cor, peso de nascimento, idade gestacional, adequação do crescimento intra-uterino e a presença de sinais, não se encontrou associação estatisticamente significante, exceto nos casos de CIV e PCA, que mostraram forte associação com a presença de sinais cardíacos e sopro. Observou-se que, dentre os $12 \mathrm{RN}$ com CIA, nove não apresentavam nenhum sinal clínico de $\mathrm{CC}$ e o diagnóstico só foi realizado porque existiam outras indicações.

\section{Discussão}

A prevalência de CC, em nosso estudo, foi de $21,8 \%$ (tab. II). Na literatura encontram-se prevalências de CC variando de 0,32 até $1,2 \%$ 5,24-27, na população geral, diferenças que poderiam ser explicadas pela falta de uniformidade metodológica e de casuística.

O estudo que mais se aproxima do nosso, tanto pela casuística como pela metodologia utilizadas, foi o de Manetti e $\mathrm{col}^{5}$, cuja prevalência foi de $1,23 \%$. Os autores sugerem que esta taxa maior de CC fosse decorrente da população estudada, pois o hospital onde foi realizado é um serviço de referência. Outra informação importante é a ocorrência de um aumento da prevalência de CC no decorrer dos anos. No período de 1975-1979, a prevalência foi de 1,15\% e, no período de 1980-1984, de 1,34\%. Os autores referem que a diferença entre os dois períodos decorre da introdução da ECO na avaliação diagnóstica, a partir de 1980, permitindo o diagnóstico mais refinado desses casos. O critério de seleção utilizado pelos autores para a realização da ECO baseou-se nos achados clínicos de sinais sugestivos de comprometimento cardíaco, critério que é diferente do utilizado neste estudo, pois o ECO foi indicado não apenas nos RN com sinais, mas em outros grupos de risco para CC. Encontrou-se em nosso estudo uma prevalência muito mais elevada do que no de Manetti e $\mathrm{col}^{5}$, provavelmente decorrente de diferenças nos critérios de seleção. Corroborando esta hipótese, observou-se neste estudo que 13 (36\%) dos RN com CC não apresentavam nenhum sinal clínico no período neonatal (tab. III).

$\mathrm{Na}$ análise da prevalência de $\mathrm{CC}$ em cada grupo de risco, verificou-se que todos os grupos apresentam taxas de prevalência maior do que as encontradas na população geral, descritas na literatura (tab. II).

No grupo I, a prevalência de $20,8 \%$ por nós encontrada, foi maior do que as descritas em outros estudos, de 2 a $15 \%{ }^{10,28}$. O tipo de CC mais freqüente nos filhos de mães com a doença foi a CIA tipo ostium secundum, em quatro dos cinco casos. Os quatro RN eram assintomáticos. Estes casos só foram diagnosticados em decorrência da mãe ter CC. Em relação à concordância entre a cardiopatia materna e a do RN, verificou-se que esta ocorreu em $40 \%$ dos nossos 
casos. No estudo de Whittemore e $\operatorname{col}^{28}$, esta concordância foi de cerca de $58 \%$.

Em relação ao grupo II, a prevalência foi de $10,7 \%$, achado este mais elevado do que na literatura, que variou de $1,7 \mathrm{a} 6,8 \%{ }^{12-14}$.

Ao analisar os RN, de acordo com a classificação do diabetes materno, segundo Priscilla-White, verificou-se que a prevalência de $\mathrm{CC}$ no grupo de $\mathrm{RN}$ filhos de mães com diabetes mellitus da classe A foi de 5,8\% (1/17), enquanto que no grupo de $\mathrm{RN}$, cujas mães são portadoras de diabetes mellitus das classes $\mathrm{B} \mathrm{e} \mathrm{C}$, foi de $18,0 \%$ (2/11). Neste último grupo, os tipos de cardiopatia foram mais graves. $\mathrm{O} 1^{\circ}$ caso, caracterizado por: CIV múltiplas, CIA tipo ostium secundum, PCA e hipertrofia de VD; o $2^{\circ}$, por dupla via de saída de VD, PCA, CIA tipo ostium secundum e CIV.

Estes nossos achados vêm corroborar a hipótese de que a $\mathrm{CC}$, assim como as demais malformações congênitas, no filho de mãe diabética, deve estar mais relacionada às alterações metabólicas graves que ocorrem no início da gestação, durante o período de organogênese ${ }^{29,30}$ do que a outros fatores.

No grupo III, constituído pelos RN com peso de nascimento $<1500 \mathrm{~g}$ e que não se enquadravam em nenhum dos outros grupos, encontrou-se uma prevalência de CC de $11,1 \%$. Como existiam vários $\mathrm{RN}$ com peso $<1500 \mathrm{~g}$ nos outros grupos estudados, optou-se por analisá-los conjuntamente, discutindo a associação entre peso de nascimento e $\mathrm{CC}$, posteriormente.

O grupo IV apresentou a prevalência mais elevada, de $40,7 \%$. Dentre os estudos consultados, a maioria estudou a associação entre a CC e a malformação extracardíaca, encontrando taxas que variavam de 25 a $45 \%{ }^{17,18}$. Poucos trabalhos consideraram o reverso, isto é, a prevalência de CC nas crianças portadoras de malformações extracardíacas, como analisado neste estudo. Numa revisão sobre o assunto, Copel e col $^{31}$ encontraram 12,2\% de CC nos RN com malformações extracardíacas. Estudos mais recentes de ECO fetal ${ }^{9,32}$ encontraram taxas de CC ao redor de $11 \%$ nos casos de fetos malformados.

De acordo com o tipo de malformação extracardíaca, estudaram-se cinco casos de cromossomopatia, sendo quatro de síndrome de Down e um de síndrome de Patau. Em todos os casos detectou-se CC. Nossos achados concordam com os dados de literatura ${ }^{17,33,34}$.

Analisaram-se sete casos de malformação renal, encontrando-se dois $(28,5 \%)$ de CC. Dentre os seis de malformações de sistema nervoso, detectou-se um $(16,6 \%)$ de CC e, nos RN com malformações músculo-esqueléticas, três $(42,8 \%)$ com CC nos sete examinados. Apesar do número pequeno de $\mathrm{RN}$ examinados por tipo de malformação, nossos dados aproximam-se dos encontrados na literatura.

Quando analisou-se o grupo V, obteve-se uma prevalência de CC de 26,8\% (tab. II). Os tipos de CC mais freqüentes foram: PCA em 27,2\%, dois casos de CIA e dois de associação de defeito do septo atrial e ventricular, numa porcentagem de $18,1 \%$ cada um, e um caso de CIV isolada, tetralogia de Fallot, AVC e cardiopatia complexa.
É importante observar que 30 RN do grupo V apresentavam sinais sugestivos de comprometimento cardíaco, não associados a cardiopatia congênita. Segundo Moss ${ }^{19}$, o sopro cardíaco no período neonatal pode ser consequiência de turbulência gerada nas artérias pulmonares periféricas, em decorrência do estreitamento desproporcional presente na luz desses vasos, simulando uma estenose de artérias pulmonares periféricas. Geralmente, o sopro desaparece ao redor dos três meses de idade.

Analisando as outras variáveis, segundo a literatura ${ }^{26,35}$ nenhum autor encontrou associação entre CC e sexo ou cor. Em nosso estudo, não se encontrou associação estatisticamente significante entre sexo e os três tipos de cardiopatia analisados isoladamente, CIA, CIV e PCA. Nos casos de PCA, houve um predomínio do sexo masculino (3:1), que não foi significante.

Vários estudos ${ }^{15,36,37}$ analisaram a relação entre $\mathrm{CC}$ e peso de nascimento, idade gestacional e retardo de crescimento intra-uterino, sendo que muitos encontraram associação positiva entre as variáveis, diferindo de nossos resultados, onde os RN com CC apresentaram uma distribuição homogênea pelas faixas de peso, idade gestacional e adequação de peso para a idade gestacional, sem diferenças estatisticamente significantes com os RN sem CC.

Observa-se que não houve associação estatisticamente significante entre a presença de $\mathrm{CC}$ e as idades pós-natal e pós-conceptual. Entretanto, observou-se uma associação, estatisticamente significante, entre a presença de sinais cardíacos e CC, sendo que a presença destes aumentou a probabilidade de $\mathrm{CC}$ em 3,58 vezes. Quando analisou-se apenas o sopro cardíaco, a associação com CC tornou-se mais forte, aumentando a chance de ter CC para 5,65 vezes (tab. IV). Ao analisar-se o valor preditivo positivo do sopro cardíaco, observou-se que este alcançou $42 \%$, quando analisados todos os RN que apresentavam este sinal clínico, e o valor preditivo negativo foi de $87 \%$. Quando analisou-se apenas os RN do grupo V, que apresentavam sopro cardíaco, este valor preditivo positivo diminuiu para $38 \%$, enquanto nos $\mathrm{RN}$ de outros grupos de risco e que apresentavam o sopro cardíaco, o valor preditivo elevou-se para $48 \%$. Verificou-se, portanto, que a presença deste sinal cardíaco, como indicativo de exploração ecocardiográfica, torna-se mais valioso quando presente em $\mathrm{RN}$ com uma outra indicação paraECO.

Deve-se, também, observar que 36\% dos RN com cardiopatia congênita não apresentavam nenhum sinal sugestivo de comprometimento cardíaco. Portanto, a presença de sinais cardíacos, especialmente sopro cardíaco, foi um achado importante para suspeita de CC, mas não o único.

Em relação ao valor preditivo negativo, verificou-se que foi de $93 \%$ nos $\mathrm{RN}$ do grupo $\mathrm{V}$ e, nos dos demais grupos de risco, de $86 \%$. A ausência de sopro cardíaco em RN que não pertenciam a nenhum outro grupo de risco tornou pouco provável a presença de CC. Porém, nos pertencentes a pelo menos um dos grupos, encontrou-se $36 \%$ com CC, na ausência de sinais clínicos sugestivos de cardiopatia. Portanto, nestes $\mathrm{RN}$ a ausência de sopro não afastou a presença de CC. 
$\mathrm{Na}$ análise da OR entre os grupos estudados, verificou-se, entre os grupos de risco, aqueles com maiores probabilidades de apresentarem CC (tab. IV). Observou-se no grupo de RN portadores de malformações extracardíacas, uma probabilidade três vezes maior de ter CC, quando comparado aos demais grupos. Apesar de todos os grupos apresentarem prevalência de CC mais elevada do que a da população geral, o grupo IV foi o que obteve maior associação com CC.

Em relação à freqüência do tipo de $\mathrm{CC}$, observou-se que nossos dados diferem muito dos da literatura ${ }^{5,13,17,25,26,38,39}$. Chama a atenção a freqüência de $35,2 \%$ de CIA, fazendo-a o tipo de CC mais freqüente, que na literatura variou de 2,1 até $11,2 \%$, sendo a cardiopatia mais freqüente a CIV. Mesmo se, na análise dos dados, classificar-se como CIV os quatro casos de cardiopatia associada, a freqüência de CIV, neste estudo, atinge $22,2 \%$, ainda abaixo da encontrada na literatura, de 24 a 50,5\%. Diferença esta, provavelmente, decorrente da metodologia utilizada nos diversos trabalhos, como seleção dos pacientes, definição de CC, método diagnóstico utilizado e tempo de acompanhamento. A maioria deles seleciona os pacientes a partir de sinais clínicos de comprometimento cardíaco e, portanto, estaria subestimando a freqüência de cardiopatias, como a CIA que, pela evolução natural da doença, mantém-se assintomática por longos períodos, sendo detectados apenas os casos mais graves. Neste estudo, a seleção dos RN foi feita a partir de grupos definidos como sendo de risco para $\mathrm{CC}$, independente da presença de sinais. Observou-se que, dos 12 casos de CIA, apenas três apresentavam sinais de comprometimento cardíaco, sendo os demais selecionados para a realização de ECO por pertencerem a outros grupos de risco (tab. V). Alguns dados de literatura ${ }^{13,38}$ vêm corroborar a hipótese de que a frequiência de CIA está subestimada pela forma de seleção dos pacientes. Dois artigos ${ }^{13,38}$ verificaram que parte dos casos de CIA foi diagnosticada apenas através dos achados de necropsia (68\% e $30 \%$, respectivamente).

A freqüência das cardiopatias complexas foi baixa, de acordo com a literatura. Mas é importante observar nestes dados que estas sempre estiveram associadas com sinais cardíacos de aparecimento precoce e pertenciam aos grupos de risco, como o diabetes materno das classes $\mathrm{B}$ e $\mathrm{C}$ de Priscilla-White e malformações extracardíacas, especialmente as cromossomopatias.

Dentre as variáveis estudadas, as únicas que apresentaram associação estatisticamente significante com a CIV e a PCA foram a presença de sinais cardíacos e o sopro cardíaco. Os quatro casos de CIV apresentaram manifestações clínicas, caracterizadas pela presença de sopro cardíaco, a partir do final da $1^{\mathrm{a}}$ semana de vida. Dos seis casos de PCA, apenas um não apresentou sinal clínico na época do diagnóstico. Dentre os que apresentaram sinais cardíacos, quatro manifestaram-se com sopro cardíaco e um com crises de cianose. Estes achados concordam com os da literatura ${ }^{40}$, que descrevem o aparecimento de sinais cardíacos nestas duas cardiopatias no período neonatal.

$\mathrm{Na}$ literatura encontramos vários estudos ${ }^{15,41,42}$ que mostram um aumento do número de casos de PCA em RNPT ou com baixo peso de nascimento. Porém, trabalho recente ${ }^{11}$ não mostrou associação entre PCA e idade gestacional ou peso de nascimento em RNPT saudáveis. Salienta-se que existe uma associação estatisticamente significante entre PCA e RNPT com insuficiência respiratória, principalmente com doença de membranas hialinas. Assim, nesta casuística, em que não se incluiu RN com insuficiência respiratória pela dificuldade técnica de realização do exame, e utilizou-se a definição de Mitchell e col ${ }^{13}$, que considera PCA em RNPT apenas quando esta é detectada a partir do $14^{\circ}$ dia de vida, não se esperaria encontrar associação entre peso de nascimento ou idade gestacional. Estudou-se também a relação entre a presença de PCA e as idades pós-natal e pósconceptual, com o interesse de verificar se o fechamento do canal arterial poderia ocorrer mais tardiamente do que o encontrado na literatura e, se este fechamento teria mais relação com a idade pós-natal ou a pós-conceptual.

O diagnóstico de PCA foi feito em seis RN, sendo que três eram RNPT e três de termo. O diagnóstico de PCA nos três casos de RNPT foi feito, em um, no $14^{\circ}$ dia, em outro, na $4^{\mathrm{a}}$ semana e, no $3^{\mathrm{o}}$, na $6^{\mathrm{a}}$ semana de vida pós-natal, os três realizaram ECO, com idades pós-conceptuais de 36, 34 5/7 e 36 1/7 semanas. Em seus acompanhamentos observou-se fechamento do canal arterial em dois casos, com idade pósnatal de três e seis semanas, e quando atingiram idade pósconceptual de 38 e 37 semanas, respectivamente. $03^{\circ}$ caso manteve o canal arterial pérvio no acompanhamento ambulatorial. Estes achados de fechamento espontâneo do canal arterial em RNPT saudáveis mais tardiamente, quando atingem idades pós-conceptuais próximas do termo, sugerem que o fechamento do canal arterial poderia estar mais relacionado com a idade pós-conceptual do que com a pósnatal. Esta suposição merece um estudo mais profundo com o acompanhamento ecocardiográfico dos RNPT até que tenham atingido a idade pós-conceptual de 37 ou 38 semanas.

Nossos resultados permitem chegar às seguintes conclusões: a prevalência de CC nos RN estudados foi muito maior do que a encontrada na literatura para a população geral de RN; a prevalência de CC em cada grupo de risco foi mais elevada do que a encontrada na literatura, sendo que a prevalência no grupo IV, foi a mais elevada; não encontramos associação estatisticamente significante entre $\mathrm{CC}$ e as variáveis: sexo, cor, peso de nascimento, idade gestacional, adequação de crescimento intra-útero, idade pós-natal e idade pós-conceptual. Encontramos associação entre $\mathrm{CC}$ e a presença de sinais clínicos e sopro cardíaco; entre os grupos de risco, o grupo IV, constituído por RN com malformações extracardíacas, foi o que mostrou maior associação com CC. A chance de CC neste grupo foi três vezes maior do que no restante dos RN estudados; a coexistência de sinais cardíacos e mais uma indicação de ECO elevam a chance de $\mathrm{CC}$ em 3,58 vezes em relação ao restante; na presença de sopro cardíaco e outra indicação de ECO, a probabilidade foi 5,65 vezes maior do que no restante dos RN estudados; a CIA foi a cardiopatia mais freqüente no grupo estudado e $75 \%$ dos casos não apresentaram nenhum sinal 
cardíaco; não foi encontrada relação entre o tipo de CC e os grupos de risco; foi encontrada associação significante entre CIV e PCA com presença de sinais cardíacos, especialmente o sopro cardíaco.

Finalmente, recomendamos a realização de ECO, no período neonatal, em RN pertencentes aos grupos de risco, procedimento que deve ser sistemático na assistência, mesmo reconhecendo que envolve consideráveis recursos materiais e humanos, pois, nossos dados mostraram que esses grupos têm uma prevalência bem mais elevada do que a da população geral e que $36 \%$ dos casos de CC não apresentavam nenhum sinal clínico, só tendo sido detectados pela ECO.

\section{Referências}

1. Castilla E - Documento final: XXI Reunion Anual do Estudo Colaborativo Latino Americano de Malformações Congênitas. UFRJ. Rio de Janeiro, Brasil, 1990.

2. Nazer J, Cifuentes L, RuizG - Incidencia de 50 malformaciones congenitas em 3 maternidades chilenas participantes del Estudio Colaborativo Latinoamericano de Malformaciones Congenitas (ECLAMC) periodo 1982-1988. Ver Chil Pediatr 1991; 62: 37-44.

3. Ramos JLA, Laurindo VM, Vaz FAC et al - Malformações congênitas: estudo prospectivo de dois anos em três maternidades de São Paulo. Pediat 1981; 3: 20-8.

4. Gurleen SK, Linsey AD - Screening for congenital heart disease prenatally. Results of a $21 / 2$ year study in the South East Thames region. Brit J Obstet Gynaec 1992; 99: 220-5

5. Manetti A, Pollini I, Cecchi F et al - Epidemiologia delle malformazioni cardiovasculari. III. Prevalenza e decorso in 46895 nati vivi alla maternitá di Carregi, Firenze, nel periodo 1975-1984. G Ital Cardiol 1993; 23: 145-52.

6. Chang AC, Hanley FL, Lock JE et al - Management and outcome of low birth weight neonates with congenital heart disease. J Pediatr 1994; 124: 461-6.

7. Cullen S, Sharland GK, Allan LD et al - Potential impact of population screening for prenatal diagosis of congenital heart disease. Arch Dis Child 1992; 67: 775-8.

8. Sharland GK, Allan LD - Screening for congenital heart disease prenatally. Results of a 2 1/2 - year study in South East Thames Region. Br J Obstet Gynaecol, 1992; 99: 220-5

9. Smythe JF, Copel JA, Kleinman CS - Outcome of prenatally detected cardiac malformations. Am J Cardiol 1992; 69: 1471-4.

10. Nora JJ, Nora AU - The evolution of specific genetic and environment counseling in congenital heart disease. Circulation 1978; 57: 205-11.

11. Reller MD, Ziegler ML, Rice MJ, Solin RC, Mcdonald RW - Duration of ductal shunt in healthy preterm infants: an echocardiographic color flow doppler study. J Pediatr 1988; 112: 441-6.

12. MillerE, Hare JW, Cloherty JP et al - Eluated maternal hemoglobin $A_{1 c}$ in early pregnancy and major congenital anomalies in infants of diabetic mothers. NEngl J Med 1981; 304: 1331-4.

13. Mitchell SC, Korones SB, Berendes HW - Congenital heart disease in 56109 births - incidence and natural history. Circulation 1971; 43: 323-32.

14. Rowland TW, Hubbell JP, Nadas AS - Congenital heart disease in infants of diabetic mothers. J Pediatr 1960; 83: 815-35.

15. Kenna AP, Smithells RW, Fielding DW - Congenital heart disease in Liverpool: 1960-69. Q J Med 1975; 44: 17-44.

16. Levy RJ, Rosenthal A, Fyler DC et al - Birthweight of infants with congenital heart disease. Am J Dis Child 1978; 132: 249-54.

17. Ferencz C, Neill CA, Boughman JA et al - Congenital cardiovascular malformations associated with chromosome abnormalities: an epidemiologic study. J Pediatr 1989; 114: 79-86.

18. Wallgren EI, Landtman B, Rapola J - Extracardiac malformations associated with congenital heart disease. Eur J Cardiol 1978; 7: 15-24.

19. Moss AJ - Clues in diagnosing congenital heart disease. West J Med 1992; 156 : 392-8.

20. Xu M, McHaffie DJ - Nonspecific systolic murmurs: an audit of the clinical value of echocardiography. NZMed J 1993; 106: 54-6.

21. Ramos JLA - Avaliação do crescimento intra-uterino por medidas antro- pométricas do recém-nascido. Tese doutoramento. Faculdade de Medicina, USP São Paulo, 1983

22. Fletcher RH, Fletcher SW, WagnerEH - Epidemiologia clínica-bases científicas da conduta médica. Porto Alegre: Artes Médicas, 1991.

23. Internacional Society of Cardiology - Classification of Heart Disease in Childhood. Groningen: VRB Offsetdrukkij, 1970.

24. Ferencz C, Rubin JD, McCarter RTJ et al -Congenital heart disease: Prevalence at livebirth. The Baltimore - Washington Infant Study. Am J Epidem 1985; 121 : 31-6.

25. Fixler DE, Pastor $\mathrm{P}$, Chamberlin $\mathrm{M}$ et al - Trends in congenital heart disease in Dallas county birth 1971-1984. Circulation 1990; 81: 137-42.

26. Laursen HB - Some epidemiological aspects of congenital heart disease in Denmark. Acta Paediatr Scand 1980; 69: 619-24.

27. Martin GR, Perry LW, Ferencz C - Increased prevalence of ventricular septal defect: epidemic or improved diagnosis. Pediatrics 1989; 83: 203.

28. Whittemore R, Hobbins JC, Engle MA - Pregnancy and its outcome in women with and without surgical treatment of congenital heart disease. Am J Cardiol 1982; 50: 641-51.

29. Coustan DR, Felig P - Diabete melito. In: Burrow GN, Ferris TF, ed Complicações Clínicas na Gravidez. São Paulo: Roca, 1989; 37-70.

30. Sadeck LSR, Leone CR, Ramos JLA et al - Síndrome de regressão caudal (SRC) em filho de mãe diabética pós-pancreatectomia. J Pediatria 1985; 58: 211-5.

31. Copel JA, Pilu G, Kleinman CS - Congenital heart disease and extracardiac anomalies: associations and indications for fetal echocardiography. Am J Obstet Gynecol 1986; 154: 1121-32.

32. Friedman AH, Copel JA, KleinmanCS - Fetal echocardiography and fetal cardiology: indications, diagnosis and management. Semin Perinatol 1993; 17: 76-88.

33. Bhatia S, Verma IC, Shrisvastava S - Congenital heart disease in Down' syndrome: an echocardiographic study. Indian Pediatr 1992; 29: 1113-6.

34. Bulbul ZR, Rosenthal D, Brueckner M - Genetic aspects of heart disease in the newborn. Semin Perinatol 1993; 17: 61-75.

35. Fyler DC-Report of the New England regional infant cardiac program. Pediatrics 1980; 65: 375-461.

36. Francannet $\mathrm{C}$, Lancaster PA, Pradat $\mathrm{P}$ et al - The epidemiology of three serious cardiac defects. A joint study between five centres. Eur J Epidemiol 1993; 9: 60716.

37. Rosenthal GL, Wilson PD, Boughman JA, Ferencz C - Birth weight and cardiovascular malformations: a population-based study. The Baltimore-Washington Infant Study. Am J Epidemiol 1991; 133: 1273-81.

38. Bound JP, Logan WFWE - Incidence of congenital heart disease in Blackpool 1957-1971. Br Heart J 1977; 39: 445-50.

39. Dolara A, Manetti A, Cicchi F et al - Epidemiologia delle malformazioni cardiovascolari. II) Follow-up di 337 nati nel periodo 1975-80 alla maternità di Firenze. G Ital Cardiol 1981; 11: 713-18.

40. Bustamante LNP, Mazzieri R, Ebaid M- Cardiopatias congênitas. In: Marcondes E, ed - Pediatria Básica. São Paulo: Sarvier, 1991; 1593-606.

41. Levin DL, Stanger P, Kitterman JA et al - Congenital heart disease in low birth weight infants. Circulation 1975; 52: 500-3.

42. Smith DRS, Cook DH, Izukawa T et al - Patent ductus arteriosus in infants of low birth weight. Can Med Assoc J 1980; 123: 739-43. 